\title{
Grupos vulnerables y margen de apreciación nacional ${ }^{1}$
}

\section{Disadvantaged Groups and Doctrine of Margin of National Appreciation}

\author{
Jaime Gajardo Falcón ${ }^{2}$ \\ Universidad Diego Portales (Chile) \\ ORCID: https://orcid.org/0000-0002-9530-9937
}

Recibido: 27-12-2019

Aceptado: 07-06-2020

\section{Resumen}

Desde la sentencia de la Corte Interamericana de Derechos Humanos (Corte IDH) en el "caso de las personas dominicanas y haitianas con República Dominicana" se ha venido discutiendo con mayor intensidad si la Corte IDH debiera comenzar a utilizar la doctrina del margen de apreciación en el espacio interamericano. En esta dirección escriben autores que discuten sobre la autoridad y legitimidad de la Corte IDH -y de los tribunales internacionales en general-para resolver casos díficiles y condenar a los Estados por vulneración a los derechos humanos contemplados en la Convención Americana de Derechos Humanos (CADH) o en el respectivo tratado internacional. Otros autores abogan por la incorporación de la doctrina del margen de apreciación por parte de la Corte IDH como una forma de articular de mejor forma la comunidad política interamericana.

Luego de revisar las posiciones doctrinal al respecto sostendré que la utilización de la doctrina del margen de apreciación en los casos de tipo multicultural puede implicar la invisibilización de las tensiones existentes al interior de las comunidades nacionales, donde muchas veces la cultura y prácticas de las minorías señaladas

\footnotetext{
${ }^{1}$ El presente artículo forma parte del proyecto de investigación "Teorías de la justicia y derecho global de los derechos humanos" (referencia PID2019-107172RB-I00), financiado por el Ministerio de Ciencia e Innovación de España.

${ }^{2}$ (jaime.gajardo@mail.udp.cl). Es abogado por la Universidad de Chile. Magíster en Derecho, con mención en Derecho Público, Universidad de Chile. Máster en Gobernanza y Derechos Humanos, Universidad Autónoma de Madrid. Máster en Derecho Constitucional, Centro de Estudios Políticos y Constitucionales. Doctor en Derecho, Universidad Autónoma de Madrid. Profesor de la cátedra de Derecho Constitucional, Universidad Diego Portales. Entre publicaciones más recientes, destacan: Derechos Humanos y Multiculturalismo (Thomson Reuters, 2020); Constitucionalismo y procesos constituyentes Vol. 1 (2018) y Vol. 2 (2019), Thomson Reuters.
} 
es menospreciada por la cultura mayoritaria o hegemónica. Este es el caso de la población indígena y migrante en el contexto américano, por lo que la utilización de la doctrina del margen de apreciación en este tipo de casos puede implicar que los tribunales internacionales convaliden la vulneración de los derechos humanos de las minorías al interior de los Estados. En ese sentido, el trabajo argumentará que una aplicación limitada del margen nacional de apreciación en casos multiculturales de grupos vulnerables permite a los tribunales regionales de derechos humanos proteger a las minorías étnicas, religiosas y culturales contra la constatada discriminación interna o contra la violación de los derechos convencionales.

Palabras-clave: Corte Interamericana de Derechos Humanos, doctrina de margen de apreciación nacional, multiculturalismo, pueblos indígenas y migrantes.

\section{Abstract}

Since the judgment of the Inter-American Court of Human Rights in the "case of Dominican and Haitian people with the Dominican Republic" it has been discussed with greater intensity if the Inter-American Court should begin to use the doctrine of the margin of appreciation in the inter-American space. At this address writers write about the authority and legitimacy of the InterAmerican Court -and international courts in general- to resolve difficult cases and condemn states for violation of human rights contemplated in the American Convention on Human Rights or in the respective international treaty. Other authors advocate the incorporation of the doctrine of the margin of appreciation by the Inter-American Court as a way to better articulate the inter-American political community.

After reviewing the doctrinal positions in this regard, I will argue that the use of the doctrine of the margin of appreciation in cases of multicultural type may imply the invisibility of existing tensions within national communities, where many times, the culture and practices of The minorities indicated are belittled by the majority or hegemonic culture. This is the case of the indigenous and migrant population in the American context, so the use of the margin of appreciation doctrine in this type of cases may imply that international courts validate the violation of the human rights of minorities within of the States. In that sense, the work will argue that a limited application of the national margin of appreciation in multicultural cases of vulnerable groups, allowing regional human rights courts to protect ethnic, religious and cultural minorities against the established internal discrimination or against the violation of the rights protected in the American Convention of Human Rights.

Key-words: Inter-American Court of Human Rights, Doctrine of Margin of National Appreciation, Multiculturalism, Indigenous and Migrant Peoples. 


\section{Grupos vulnerables e interpretación de los derechos humanos}

El fenómeno multicultural plantea una serie de dificultades para acomodar la diversidad cultural a los modelos clásicos del Estado constitucional y a las instituciones internacionales que protegen los derechos humanos, dentro de las que se encuentran los tribunales regionales como la Corte IDH y el Tribunal Europeo de Derechos Humanos (TEDH). Sin embargo, las propuestas que se han formulado entendidas contextualmente pueden ayudar a la superación de dichos inconvenientes.

Concordando con lo apuntado por Ignacio Gutiérrez, considero que el desafío del multiculturalismo depende, a la postre, de las posibilidades abiertas a la plena participación de todos, principalmente, de aquellos que han sido histórica y estructuralmente excluidos del proceso democrático y, por ende, de la deliberación política ${ }^{3}$. Para ello, junto con fórmulas nuevas que se incorporen a la democracia representativa que permitan la inclusión de perspectivas sociales de los grupos etno-culturales desaventajados ${ }^{4}$, también puede jugar un papel importante la incorporación de la perspectiva multicultural en la interpretación de los casos difíciles que conozcan los tribunales nacionales e internacionales que involucren derechos humanos 5 .

\footnotetext{
${ }^{3}$ Cf. Gutiérrez 2007: 20. Asimismo, véase: Tully 2007: 22; Pentassuglia 2009: 195-198.

${ }^{4}$ En ese sentido, Denninger señala que: "El camino de la integración mediante el Derecho es rico en presupuestos. Exige el reconocimiento de todos los miembros de la sociedad como libres e iguales, tanto el reconocimiento recíproco de los miembros entre sí como también ese reconocimiento en la relación de los individuos con el Estado. Y exige ante todo acordar conjuntamente y reconocer reglas de procedimiento, que han de ser respetadas por todos, para la generación de "Derecho". No significa otra cosa el consenso democrático fundamental que idealmente precede a todos los esfuerzos en favor de un "Derecho correcto" desde el punto de vista material: el acuerdo sobre la procedimentalización que ha de seguirse para la creación del Derecho, por ejemplo mediante decisiones mayoritarias de los órganos "representativos" convocados al efecto. Si este acuerdo no se logra, el proceso de integración no saldrá de una situación de guerra civil latente, aunque no se llegue a uso alguno de la fuerza". Cf. Denninger, 2007: 37-38.

5 Denninger, luego de analizar dos casos alemanes (uno sobre un grupo Musulmán y otro Testigo de Jehová), señala que es necesario un criterio flexible de interpretación de las cláusulas democráticas, señalando que: "Para la pacífica convivencia democrática de los ciudadanos, dice el Tribunal Constitucional, es suficiente con el acatamiento de la Ley que prestan por principio, con el respeto a los principios constitucionales fundamentales (art. 79.3 de la Ley Fundamental), a los derechos fundamentales de los demás y a los principios fundamentales que inspiran en un régimen de libertad el Derecho eclesiástico y la regulación jurídica de la religión. Lo decisivo estaría en el comportamiento efectivo de los titulares del derecho fundamental, no en investigar sus convicciones. La Ley Fundamental no exige una lealtad al Estado que vaya más allá de esto. La democracia liberal espera ciertamente de los ciudadanos que participen las elecciones políticas en medida suficiente, pero con buenos motivos no ha configurado dicha "expectativa" como deber jurídico, por tanto con carácter vinculante”. Denninger, 2007: 39-41. También puede jugar un papel importante el principio de deferencia legislativa, cuando en la deliberación política han participado los grupos etno-culturales. En ese sentido y sobre la decisión del Tribunal Constitucional Alemán, en el "caso del velo", véase: Denninger, 2007: 42-43. En el debate de la revisión judicial en los Estados Unidos, John Hart Ely ha señalado que la intervención judicial es legítima y no antidemocrática cuando corrige un proceso político defectuoso por no considerar la participación de minorías o grupos que se encuentran al margen de la deliberación política. Al respecto, véase: Ely 1980: 136. En el mismo sentido, pero
} 
En general, las constituciones políticas o los convenios internacionales tales como la CADH o el Convenio Europeo de Derechos Humanos (CEDH) no reconocen derechos humanos que incorporen derechos de identidad grupal o protejan expresamente a ciertas minorías culturales ${ }^{6}$, por lo cual las consideraciones de la óptica multicultural han surgido principalmente en el momento de la interpretación de los derechos ${ }^{7}$, siendo los límites y la legitimidad de la revisión judicial un asunto largamente discutido en el debate académico $^{8}$.

En la práctica interpretativa los tribunales constitucionales e internacionales de Derechos Humanos han oscilado vacilantemente con sus sentencias, desde la búsqueda de la asimilación cultural al relativismo cultural. Como punto intermedio y buscando superar esas lógicas de entendimiento del fenómeno multicultural, Dieter Grimm plantea una solución contextual al problema, que busca la integración cultural y se encuentra cercana a los planteamientos del liberalismo multicultural ${ }^{9}$.

llevando la propuesta de Ely a los tribunales regionales de protección de los derechos humanos, véase: Popellier y Van de Heyning 2017: 5-23.

${ }_{6}$ En América Latina, con el fenómeno de la constitucionalización de los derechos de los pueblos indígenas, se ha incorporado la identidad grupal y protección a determinados grupos en la configuración de especiales derechos fundamentales. Siendo paradigmático en ello la Constitución del Estado Plurinacional de Bolivia de 2009. Otro ejemplo interesante, es el artículo 27 de la Constitución canadiense que señala que toda la interpretación de la misma debe concordar con el objetivo de promover el mantenimiento y la valoración del patrimonio multicultural de los canadienses. Dicho artículo ha permitio generar la herramienta hermenéutica de la "acomodación razonable" la que "supone la obligación de adoptar todas las medidas apropiadas y razonables para evitar que discriminaciones indirectas puedan suponer una vulneración al principio de igualdad en ámbitos laborales, educacionales, sanitarios, etc.". Cf. Caicedo 2015: 44.

${ }^{7}$ En ese sentido, Dieter Grimm señala que "[E]1 derecho fundamental a la libertad de asociación no puede incorporar tal función; al margen de que está reservado a los nacionales [alemanes], no proporciona ningún argumento en el ámbito preciso de la garantía de la identidad en el seno de un ordenamiento jurídico determinado por otros valores y tradiciones. Asegura ante todo el derecho de los individuos a asociarse con otros para los fines que ellos mismos determinen. La protección se extiende ciertamente también a la asociación, pero no permite ampliar su marco jurídico de acción: también las asociaciones deben respetar lo que está mandado o prohibido con carácter general”. Cf. Grimm, 2007: 57.

${ }^{8}$ Para una defensa en términos teóricos de la revisión judicial debido a la textura abierta y la idea de que las normas constitucionales deben tener una "lectura moral", entre otros, véase: Dworkin 1984 y Perry 2007. Para una crítica de la revisión judicial centrada en la idea de que los desacuerdos morales se deben resolver en el debate democrático en el parlamento y no en sede judicial, entre otros, véase: Waldron 2005; Atria 2016: 219-342. Para una defensa de la revisión judicial de tipo procedimental o que busque corregir los defectos del proceso democrático en términos de inclusión de personas y grupos que se encuentran al margen de la deliberación política, véase: Ely 1980 y Habermas 1998. Por su parte, para Gaetano Pentassuglia y James Tully, en sociedades multiculturales, la revisión judicial tiene un doble propósito. En primer lugar, generar correcciones en el proceso democrático de elaboración normativa, dando lugar a las perspectivas de individuos y grupos culturales que se encuentran excluídos del proceso político. En segundo lugar, la revisión judicial debe ser un mecanismo que permita ampliar el diálogo multicultural. Al respecto, véase: Tully 2007: 22; Pentassuglia 2009: 196-198. Para una crítica de la función judicial de los tribunales internacionales de protección de los derechos humanos, véase: Atria 2014: 467-484.

${ }^{9}$ Si bien se está refiriendo a la integración de los inmigrantes, mutatis mutandis, su idea es utilizable para los propósitos del presente trabajo. Grimm, 2007: 60-61. 
Para Dieter Grimm, con quien concuerdo, la óptica multicultural hay que integrarla a los criterios de ponderación ${ }^{10}$; conduce siempre a respuestas orientadas al caso concreto y al procesar los problemas individualmente permite aminorar el material conflictivo y aumentar la posibilidad de encontrar soluciones susceptibles de ser aceptadas por mayores sectores de la sociedad ${ }^{11}$.

En el sentido de la interpretación contextual, una medida procesal que se ha ensayado, y que puede tener importantes consecuencias prácticas, es la inversión de la carga de la prueba: que cuando un grupo o individuo miembro de un grupo desaventajado solicita protección de sus derechos fundamentales por sentirse agraviado por algún acto de autoridad pública o de algún privado, sean estos últimos los que tengan la carga de la prueba. Así, la inversión de la misma permitiría evaluar de mejor forma las razones para la actuación pública y someterla a una evaluación más severa. Este tipo de razonamiento, es más común en la interpretación y ponderación que realiza la Corte IDH a diferencia del TEDH, que es más reacio a invertir la carga de la prueba a favor de los peticionarios y de hecho presenta mayor deferencia, a través de la doctrina del margen de apreciación, a la actividad de los organismos públicos nacionales ${ }^{12}$.

De forma más concreta, María José Añón plantea criterios interpretativos complementarios, ya que para ella el derecho antidiscriminatorio no ha sido suficiente para lograr superar la "discriminación estructural" en la que se encuentran ciertos individuos y grupos culturales ${ }^{13}$. El enfoque de la discriminación estructural como criterio interpretativo sitúa el origen del estado de privación en el que se encuentran la mayoría de grupos marginados en los procesos sociales y en las prácticas culturales que definen su estatus, condicionando sus opciones vitales. Así, plantea que

la desigualdad estructural es algo distinto de la idea de desventajas transitorias y fortuitas que podrían ser el producto de la mala suerte o derivar de decisiones

${ }^{10}$ Cf. Grimm, 2007: 60-68.

${ }^{11}$ En un sentido similar y abogando por una interpretación contextualizada, véase: Álvarez 2014: 179-212. Asimismo, Will Kymlicka señala que: "Some critics accept that the justice of multicultural demands must be evaluated on case-by-case basis, and so focus on the potential injusties of particular multicultural proposals in particular contexts, rather than making global claims about the inherit injustice of group-specific policies. These sorts of context-specific arguments are, I think, essential, and reflect real progress in the debate. At the level of particular cases, the debate focuses, not on whether multiculturalism is right or wrong in principle, but rather on a range of more practical issues about the distribution of the benefits and burdens of specific policies". Cf. Kymlicka 1999: 114.

${ }^{12}$ La jurisprudencia del TEDH ha mostrado una tendencia a invocar la doctrina del margen de apreciación para tener una mayor deferencia con la actividad estatal. Para un ejemplo de ello véase: Gajardo Falcón 2015: 769-783. Sin embargo, hay algunos ejemplos de un incipiente uso de la interpretación contextual por parte del TEDH; al respecto, véase: Gajardo Falcón 2014: 273-286. Asimismo, el TEDH ha invertido la carga de la prueba en casos de "discriminación múltiple", por ejemplo en la sentencia del caso "B.S.C. c. España" conocido como "Solomon c. España". Al respecto véase: STEDH (2012). B.S.C c. España. Para un análisis de la sentencia, véase: Abril 2013: 309-326.

${ }^{13}$ Para una crítica a los presupuestos formales del derecho antidiscriminatorio, véase: Barrère y Morondo 2011: 15-42. 
individuales desacertadas. En este sentido, el enfoque desde la discriminación estructural puede ser entendido como un criterio interpretativo de apoyo de la discriminación indirecta, porque para comprender el sentido (...) encontramos razones de mayor peso o más justificadas si el argumento que aporta la discriminación indirecta se auxilia de otras razones de apoyo que se encuentran en el concepto de discriminación estructural ${ }^{14}$.

En ese sentido, el enfoque desde la discriminación estructural puede ser entendido como un estándar interpretativo adicional a la discriminación indirecta, debido a que además de las razones que ésta proporciona le sumamos o le incorporamos otras razones de apoyo (más generales y de mayor peso) que se encuentran en el concepto de discriminación estructural ${ }^{15}$. Los criterios interpretativos complementarios son los siguientes: la perspectiva de la discriminación estructural y el enfoque de la interseccionalidad ${ }^{16}$. Para la autora, el concepto de discriminación estructural es una propuesta doctrinal dirigida a redefinir el concepto jurídico tradicional de discriminación y a dar cabida en su seno a la noción de opresión intergrupal en términos sistémicos ${ }^{17}$. Así, señala que:

Con esta expresión podemos hacer referencia a aquellas situaciones de desigualdad social, de subordinación o de dominación, en las que no es posible individualizar una conducta determinada o identificar un trato al que se imputa la prohibición jurídica de discriminación, siendo, por tanto, situaciones que quedan fuera del concepto jurídico de discriminación. Es un tipo de desigualdad basada en el estatus, el poder de definición de identidades y la toma de decisiones. Se trata de dinámicas sociales reiteradas que llevan a la persistencia de estructuras de subordinación y resultados sistemáticamente desventajosos para ciertos grupos, incluso en ausencia de motivos discriminatorios explícitos amparados por el derecho. En este caso, de modo similar al supuesto de discriminación indirecta, acreditar la existencia de discriminación supone aportar datos empíricos o pruebas estadísticas que demuestren este sesgo "invisible" en la toma de decisiones ${ }^{18}$.

${ }^{14}$ Cf. Añón 2013: 147.

${ }^{15}$ Cf. Añón 2013: 147.

${ }^{16}$ Para María Caterina La Barbera, el enfoque de la interseccionalidad se refiere a los procesos complejos que se derivan de la interacción de factores sociales, económicos, políticos, culturales y simbólicos de cada contexto. La interseccionalidad es fundamental para examinar las diferentes dimensiones de la vida social que resultan distorsionadas cuando se adopta solo un eje de análisis o se le miran desde una sola perspectiva. Cf. La Barbera 2011: 2191. En ese sentido, la discriminación interseccional se daría cuando: “(...) dos o varios campos de discriminación se dan simultáneamente e interactúan potenciándose los efectos. Este efecto no se puede ver haciendo un análisis separado de cada uno de los campos, sino que debe verse en su contexto dado que la discriminación observada solo puede ser apreciada en su totalidad". Cf. Abril 2013: 321.

${ }^{17}$ Cf. Añón 2013: 147.

${ }_{18}$ Añón 2013: 148. 
Asimismo, acertadamente y tomando en consideración lo anterior, la autora señala que:

(...) desconocer u ocultar los patrones de discriminación estructural puede llevar a presentar como una decisión libre, algo que, en realidad, no es sino una preferencia adaptativa o una decisión marcada por un estado de necesidad que invalida la presunción de consentir de manera libre e informada ${ }^{19}$.

De lo anterior, como una práctica de desventaja masiva o estructural que puede reducir significativamente la autonomía relacional de las personas, pone como ejemplo el caso D.H. c. la República Checa, resuelto por el TEDH en el año 2007 y que trata sobre el consentimiento formal que daban los padres de hijos gitanos para que estos asistieran a escuelas donde sólo iban niños de etnia romaní ${ }^{20}$. En ese sentido, propone como criterio interpretativo la idea del umbral, donde el consentimiento de los padres es importante, pero sólo más allá de un umbral que marcaría la línea por debajo de la cual se puede ocasionar un daño inasumible a los niños. Comentando esto, María José Añón señala que el problema es definir dónde se sitúa el umbral, aunque es algo que se debería establecer a través de una deliberación política amplia y sobre cada caso particular ${ }^{21}$.

El enfoque de la interseccionalidad debería entenderse de forma complementaria al anterior y tiene por objeto:

(...) fortalecer los argumentos que sirven para identificar los patrones de discriminación de las normas que son consideradas como discriminatorias. Fundamentalmente porque es un punto de vista que permite dar cuenta de estructuras de discriminación en los sistemas de opresión o subordinación. La perspectiva que proporciona la discriminación entrecruzada o interseccional consiste precisamente en hacernos reflexionar sobre tres aspectos importantes:

a) cuestionar los modos dominantes de pensar la discriminación, al considerar que los sistemas jurídicos sólo parcialmente reconocen las estructuras de discriminación; b) explicitar cómo los sistemas jurídicos esbozan sus propias normas antidiscriminatorias a través de tipos y paradigmas de trato desigual que se muestran incapaces de identificar correctamente las categorías específicas de quienes son discriminados; c) determinar la desventaja como forma de identificación de categorías o clases de sujetos ${ }^{22}$.

Así, en relación con los criterios y enfoques mencionados, considero relevante señalar lo siguiente: en primer lugar, ambos criterios de interpretación

\footnotetext{
19 Ídem.

20 Añón 2013: 149. Para un análisis crítico de estas soluciones jurisprudenciales, véase: Carmona 2014: 587-612.

${ }^{21}$ Cf. Añón 2013: 150.

22 Añón 2013: 151.
}

Araucaria. Revista Iberoamericana de Filosofía, Política, Humanidades y Relaciones Internacionales, año $23, \mathrm{n}^{\circ} 46$. Primer cuatrimestre de 2021. Pp. 613-629. ISSN 1575-6823 e-ISSN 2340-2199 https://dx.doi.org/10.12795/araucaria.2021.i46.30 
se entienden mejor, en relación con las diferencias culturales o grupos étnicos o nacionales, si se aceptan los presupuestos teóricos del liberalismo cultural o del interculturalismo y, además, requerirían la aceptación o el reconocimiento de cierto tipo de derechos de grupo. A mi juicio, ello es complejo, ya que uno de los aspectos más discutidos en el plano multicultural son los derechos colectivos o de grupo. En ese sentido, la idea de interpretación contextualizada me parece más fácil de ser compartida por un espectro más grande, ya que puede funcionar sin que exista una total necesidad de aceptar teóricamente los derechos de grupo.

En segundo lugar, la principal limitación de las propuestas revisadas tiene que ver con lo general de las mismas y la dificultad que pueden tener para una aplicación efectiva y uniforme. En ese sentido, la idea de la interpretación contextualizada necesita de un mayor refinamiento de sus características y aspectos, ya que en términos tan generales puede correr el riesgo de que pese a ser un concepto necesario se diluya en una utilización dispar de la misma. En ese sentido, un primer paso puede ser la utilización de la inversión de la carga de la prueba cuando quien solicita protección de sus derechos fundamentales sea un grupo que claramente pueda ser identificado como desaventajado en términos culturales o un miembro de dicho grupo en sus relaciones externas. En las relaciones internas de los grupos se debe tener mucho cuidado, teniendo presente la idea de Kymlicka sobre el límite del liberalismo cultural, en cuanto a protecciones externas versus restricciones internas.

Por su parte, los criterios complementarios que plantea Añón, si bien se encuentran más desarrollados doctrinariamente, aunque no lo señala expresamente, pareciera que utiliza la idea de grupo desaventajado de Iris Young $^{23}$, que ha sido criticada por ampliar desmesuradamente el concepto de grupo, posible de aplicarse a muchas colectividades y, por ende, diluyendo la idea misma de grupo desaventajado. Aunque una forma de salvar dicha crítica sea la reducción de su campo de aplicación (por ejemplo, a los grupos culturales), Añón no profundiza lo suficiente sobre los problemas de definición, alcance, permanencia y estabilidad de un grupo desaventajado, aspectos necesarios para aplicarles una deferencia interpretativa.

La interpretación contextualizada y los criterios complementarios que plantea Añón me parece que, con su debido refinamiento, pueden ser muy útiles en la comprensión de grupos desaventajados que son bivalentes (en la terminología que ha utilizado Nancy Fraser ${ }^{24}$ ), esto es, grupos que tienen una condición de desaventajados tanto en un plano económico como identitario. En América Latina, dicha característica la encontramos en los pueblos indígenas, donde la combinación de ambos criterios puede dar paso a las necesidades de

\footnotetext{
${ }^{23}$ Véase, Young 2000.
}

24 Véase, Fraser 2006. 
reconocimiento y redistribución que requiere este tipo de colectivos para gozar de un pleno ejercicio de la igualdad material.

Llevando las ideas señaladas a la práctica, la Corte Constitucional colombiana ha generado una serie de criterios para interpretar la Constitución y, al mismo tiempo, ser deferente con la autonomía y los derechos de los pueblos indígenas de dicho país, en especial al resolver casos que tienen relación con los límites de competencia de las jurisdicciones indígenas ${ }^{25}$. En ese sentido, para la Corte Constitucional estos casos deben ser resueltos a través de la ponderación de los principios en colisión y siendo deferentes con la autonomía de las comunidades indígenas ${ }^{26}$. Sin embargo, para la Corte Constitucional la autonomía de las comunidades indígenas conoce límites, aunque la carga de la prueba la tiene el que quiera restringir la autonomía de las comunidades, debiendo demostrar que: 1) busca la protección de un intéres de mayor entidad que la diversidad cultural y étnica; 2) que se está escogiendo la alternativa que menos impacta en la autonomía de las comunidades indígenas ${ }^{27}$.

Asimismo, para la Corte Constitucional colombiana los conflictos entre la jurisdicción indígena y la justicia nacional deben resolverse bajo los siguientes parámetros: 1) análisis caso a caso teniendo especial consideración por el contexto del mismo; 2) la autonomía de las comunidades y la conservación de sus usos y costumbres tienen una relación directamente proporcional; 3) los derechos fundamentales son el límite en las relaciones al interior de las comunidades; 4) las normas imperativas, por regla general, priman sobre los usos y costumbres de las comunidades indígenas; 5) las normas dispositivas, por regla general, ceden ante los usos y costumbres de las comunidades indígenas ${ }^{28}$.

Además, la Corte Constitucional colombiana establece reglas para realizar la labor de adjudicación y resolver los casos en que están en juego los derechos fundamentales de los miembros de las comunidades indígenas. Así, en primer lugar establece una serie de criterios para analizar el contexto del caso, esto es: 1) determinar la cultura que se encuentra involucrada; 2) el grado de aislamiento o integración de la cultura en cuestión con la sociedad mayoritaria; 3 ) el grado de afectación de los derechos individuales de los miembros de la comunidad indígena ${ }^{29}$. Luego, como segundo paso, determina un conjunto de derechos fundamentales que operan como límite concreto a la autonomía indígena: 1) el derecho a la vida; 2) la prohibición de la esclavitud; 3) la prohibición de la tortura; 4) la garantía del debido proceso y el derecho a la defensa; 5) la

25 Véase, Roa 2014: 101-121. Estos criterios los ha ido construyendo la Corte Constitucional colombiana en los siguientes fallos: 1) T-254 de 1994; 2) T-349 de 1996; 3) C-139 de 1996; 4) T-523 de 1997; 5) T-510 de 1998; 6) T-1127 de 2001; 7) T-048 de 2002; 8) T-001 de 2012.

${ }^{26}$ Cf. Roa 2014: 110.

27 Roa 2014: 110-111.

28 Ídem.

29 Roa 2014: 111.

Araucaria. Revista Iberoamericana de Filosofia, Politica, Humanidades y Relaciones Internacionales, año $23, \mathrm{n}^{\circ} 46$. Primer cuatrimestre de 2021. Pp. 613-629. ISSN 1575-6823 e-ISSN 2340-2199 https://dx.doi.org/10.12795/araucaria.2021.i46.30 
legalidad del procedimiento, de los delitos y de las penas; 6) la proscripción del destierro, la prisión perpetua, la confiscación y la responsabilidad objetiva; 7) la interdicción de la arbitrariedad ${ }^{30}$.

Como se puede apreciar, la Corte Constitucional colombiana nos entrega una guía para incorporar el contexto en los casos de tipo multicultural y reglas para ser deferentes con la cultura y la autonomía de los grupos étnicos y culturales. Asimismo, incluye la inversión de la carga de la prueba, llevándola a quien busca limitar la autonomía de las comunidades indígenas. Finalmente, la Corte Constitucional colombiana establece una serie de derechos fundamentales que operarían como límite absoluto a las prácticas culturales, dejando un amplio campo de derechos fundamentales en los que cabría un entendimiento diferente $\mathrm{y}$, por ende, un culturalización de los mismos.

De forma similar, la Corte IDH ha utilizado la idea del estereotipo ${ }^{31}$, desde la sentencia en el caso conocido como Campo Algodonero con México del año $2009^{32}$, para resolver casos que envuelven una discriminación estructural en contra de las mujeres ${ }^{33}$. La forma de razonamiento utilizada por la Corte IDH para establecer la existencia de un estereotipo y luego evaluar si la práctica estatal se vio influenciada por este, es una forma de decantar y aplicar concretamente los presupuestos de la discrminación estructural. Para esto la Corte IDH, en primer lugar, en el caso Campo Algodonero evidenció la existencia de un estereotipo en contra de las mujeres en el Estado de Chihuahua (México) y, en segundo lugar, comprobó que los agentes estatales no sólo actuaron conforme a él sino que son parte importante en la consolidación del mismo. Si bien la Corte IDH ha utilizado esta herramienta para llevar a la práctica la idea de discriminación estructural principalmente, en casos relacionados a los derechos de mujeres, es un buen ejemplo de cómo se podría utilizar para casos de tipo multicultural.

\section{EI margen de apreciación y la Corte IDH}

Para algunos autores, el "caso de las personas dominicanas y haitianas contra República Dominicana" era una buena oportunidad para que la Corte IDH utilizara la doctrina del margen de apreciación y no debilitara su autoridad en el

\footnotetext{
30 Ídem.

${ }^{31}$ Para Rebecca Cook y Simone Cusak un estereotipo es una visión generalizada o una preconcepción sobre atributos o características de los miembros de un grupo en particular o sobre los roles que tales miembros deben cumplir, por ejemplo en el caso de las mujeres, lesbianas o adolescentes. Al respecto, véase: Cook y Cusack 2010: 11.

32 Al respecto, véase: SCrIDH (2009). Caso González y Otras vs. México.

33 Sobre la sentencia de la Corte IDH en el caso conocido como Campo Algodonero vs. México, véanse los trabajos de Sordo (2012 y 2014).
} 
espacio interamericano ${ }^{34}$. En esta dirección escriben autores que discuten sobre la autoridad y legitimidad de la Corte IDH -y de los tribunales internacionales en general- para resolver casos díficiles y condenar a los Estados por vulneración de los derechos humanos contemplados en la CADH o en el respectivo tratado internaciona $\mathrm{l}^{35}$. Otros autores abogan por la incorporación de la doctrina del margen de apreciación por parte de la Corte IDH como una forma de articular de mejor forma la comunidad política interamericana y mantener el "necesario equilibrio entre lo común y lo particular, entre lo nacional y lo internacional; en definitiva, entre la unidad y la diversidad" 36 .

Sobre este aspecto podemos constatar que la aplicación de la doctrina del margen de apreciación en la jurisprudencia de la Corte IDH ha sido escasa ${ }^{37}$ y los autores latinoamericanos han dado razones de texto ${ }^{38}$ y sustantivas para que esta doctrina no tenga recepción en el continente americano. En ese sentido, el otrora Presidente de la Corte IDH, el Juez Cançado Trindade ha señalado, vehementemente, que:

Cómo pretender aplicarlo en un sistema en que el Poder Judicial de tantos países sufre todo tio de presiones e intimidaciones. Cómo pretender aplicarlo en un continente en que la función judicial, en tantos países sigue siendo 'compartida' por el fuero ordinario o común, y fueros militares especiales. Cómo pretender aplicarlo en ordenamientos jurídicos nacionales severamente cuestionados por su ineficacia en el combate a la impunidad. En nuestro sufrido continente -así como en la pan-Europa convulsionada de hoy- dificilmente encontraríamos la doctrina básica sobre la cual se ha erigido en las últimas décadas la doctrina del 'margen de apreciación' de los Estados en cuanto a los modos de cumplimiento de sus obligaciones convencionales en materia de derechos humanos. Siendo así,

${ }^{34}$ En ese sentido, véase: Torres Zúñiga 2016: 102.

35 Entre otros, véase: Gargarella 2016: 409-433; Verdugo y García 2012: 175-216.

${ }^{36}$ Cf. Núñez 2012: 44. Asimismo, véase: Benavides 2009: 89; Contreras 2012: 81-82; Paúl 2014 : 123-125; Martínez Estay 2014: 339-340.

${ }^{37}$ La doctrina del margen de apreciación ha sido citada por la Corte IDH en la "Opinión Consultiva sobre la propuesta de modificación a la Constitución Política de Costa Rica relacionada con la naturalización (1984)", en el caso "Herrera Ulloa con Costa Rica (2004)" y en el caso "Barreto Leiva con Venezuela (2009)". Algunos jueces la Corte IDH en sus votos particulares han utilizado la doctrina del margen de apreciación, por ejemplo, el Juez Pérez en el caso "Atala Riffo con Chile (2012)". Los casos en que la CIDH ha utilizado el margen de apreciación en su jurisprudencia, pueden ser consultados en: Martínez Estay 2014: 315-317. Sin embargo, para Natalia Torres la Corte IDH de forma implícita ha utilizado la doctrina del margen de apreciación, por ejemplo, en casos en los que ha contralado la convencionalidad de normas constitucionales. Cf. Torres Zúñiga 2016: 105-114. Por su parte Pablo Contreras realiza una sistematización de los casos en los que la Corte IDH ha utilizado un margen de apreciación en el sentido de que supervisa la decisión nacional si la restricción del derecho se encuentra prescrita en la ley, si tiene un fin legítimo o si ha sido proporcional. Al respecto, véase: Contreras 2012: 66-81.

${ }^{38}$ En ese sentido, Héctor Faúndez señala que: "La idea de que el Estado pueda ejercer un margen de apreciación en la aplicación de la Convención no se encuentra expresamente reconocida por la Convención Americana, y tiene que ser vista, si no con recelo, por lo menos con mucha precaución; sin embargo, es evidente que esta doctrina también tiene aplicación en el sistema interamericano y que es inherente a las expresiones utilizadas por algunas de sus disposiciones”. Cf. Faúndez 2004: 57.

Araucaria. Revista Iberoamericana de Filosofia, Política, Humanidades y Relaciones Internacionales, año $23, \mathrm{n}^{\circ} 46$. Primer cuatrimestre de 2021. Pp. 613-629. ISSN 1575-6823 e-ISSN 2340-2199 https://dx.doi.org/10.12795/araucaria.2021.i46.30 
no resta otro camino sino el fortalecimiento de los mecanismos internacionales de protección, complementarios de las instancias nacionales"39.

Sin embargo, las razones señaladas no parecen concluyentes para dar por descontada la aplicación de la doctrina del margen de apreciación en el SIDH. En primer lugar, el argumento de texto no es suficiente para descartar la utilización de una herramienta de hermeútica por parte de la Corte IDH, a lo más, podría resultar relevante como un límite intrínsico para su aplicación, pero no para dejarla completamente de lado $^{40}$. En segundo lugar, las razones sustantivas que da el Juez Cançado Trindade tienen relación con la constatación del defícit democrático de las sociedades latinoamericanas, siendo una razón contingente $\mathrm{y}$, por ende, que puede cambiar con el tiempo ${ }^{41}$. Es decir, si la decisión tomada en sede nacional para la configuración de un derecho o la resolucion de un caso determinado ha sido tomada en el marco de una sociedad democrática y de una deliberación profunda, ello podría habilitar a los jueces de la Corte IDH para la utilización de la doctrina del margen de apreciación ${ }^{42}$.

\section{A modo de conclusión}

Ahora bien, concordando con la idea de que la doctrina del margen de apreciación es una herramienta hermeútica útil para los tribunales internacionales y que debería ser adoptada de forma consistente por parte de la Corte $\mathrm{IDH}^{43}$, considero que una interpretación contextualizada de los derechos

\footnotetext{
${ }^{39}$ Cf. Cançado 2006: 390.

${ }^{40}$ De forma esclarecedora, Francisco Barbosa, distingue entre límites extrínsecos e intrínsecos en la actividad de adjudicación que tienen los tribunales regionales de protección de los derechos humanos. Los límites extrínsecos serían aquellos determinados a través de las obligaciones generales y los deberes de adaptación de las disposiciones estatales cuando se firman y ratifican los tratados y por parte de los condicionamientos percibidos dentro de los derechos que consagran los convenios de derechos humanos, ya sea la CADH o el CEDH. Por su parte los límites intrínsecos están dados por la utilización de la proporcionalidad como principio de corrección funcional de la jurisprudencia internacional, el que se aplica como punto de contacto entre el control judicial y el margen de apreciación nacional. Cf. Barbosa 2011: 112-125.

${ }^{41}$ Para las críticas que se le han formulado a la postura del Juez Cançado Trindade en ese sentido, véase: Contreras 2012: 61-63.

42 En ese sentido, véase: Gargarella 2016: 428-431.

${ }^{43}$ Comparto las ideas de reformulación a la doctrina del margen de apreciación que ha planteado Marisa Iglesias refiriéndose a ella en el espacio europeo, en el sentido de una versión racionalizada de la misma, la que consistiría en "(...) efectuar un balance entre los valores que dan sentido al Convenio como instrumento jurídico. Desde esta concepción, el razonamiento del TEDH se centra en examinar si la medida estatal impugnada consigue alcanzar un balance equitativo entre derechos individuales y valores democráticos, atendiendo al dualismo axiológico entre democracia y derechos que el propio convenio reconoce en su preámbulo. Aquí la argumentación del Tribunal no tiene por qué estar mediatizada por consideraciones generales acerca de su legitimidad institucional ni la voluntad estatal tiene por qué adquirir un valor independiente. Su valor será funcional al éxito del estado en conseguir este equilibrio axiológico inherente a una sociedad democrática. La finalidad de esta versión
} 
humanos en el marco de sociedades multiculturales debiera limitar el uso de la doctrina del margen de apreciación cuando los casos son de tipo multicultural. En los casos de tipo multicultural se ven involucradas minorías étnicas, culturales o religiosas que se encuentran al margen de la deliberación política por razones estructurales e históricas, teniendo una posición débil al interior de la comunidad nacional y, por ende, poca o nula incidencia en la configuración nacional de la protección de los derechos humanos.

Debido a lo anterior, la utilización de la doctrina del margen de apreciación en los casos de tipo multicultural puede implicar la invisibilización de las tensiones existentes al interior de las comunidades nacionales, donde muchas veces la cultura y prácticas de las minorías señaladas es menospreciada por la cultura mayoritaria o hegemónica. Este es el caso de la población migrante en el contexto américano (y europeo), por lo que la utilización de la doctrina del margen de apreciación en este tipo de casos puede implicar que los tribunales internacionales convaliden la vulneración de los derechos humanos de las minorías al interior de los Estados. En ese sentido, concordando con Francisco Barbosa, el límite al margen nacional de apreciación permite a los tribunales regionales de derechos humanos proteger a las minorías étnicas, religiosas y culturales contra la constatada discriminación interna o contra la violación de los derechos convencionales. Asimismo, el límite a dicha doctrina permite construir un consenso en torno a la protección de minorías dentro de un Estado determinado y adaptar los ordenamientos internos a estándares regionales o internacionales. Esto quiere decir que formulando patrones comunes de protección o estableciendo consensos interpretativos se protegen diversidades nacionales que en muchas ocasiones quedarían fuera si se utiliza el margen de apreciación debido a la inexistencia de un consenso a nivel regional ${ }^{44}$.

racionalizada, entonces, no sería simplemente la justificación de la "deferencia" al estado sino el reconocimiento equilibrado de los valores democráticos en el sistema de protección de derechos humanos en Europa". Cf. Iglesias 2016: 186.

${ }^{44}$ Cf. Barbosa 2011: 130. En ese mismo sentido, véase: Perry 2007 y Pentassuglia 2009. 


\section{Referencias bibliográficas:}

Abril, Ruth "El reconocimiento judicial de la discriminación múltiple en el ámbito europeo" en Revista de Derecho Comunitario Europeo, 44 (2013), pp. 309-326.

Álvarez Medina, Silvina "Los derechos humanos como valores plurales. Multiculturalismo, cosmopolitismo y conflictos". [En: Ruiz Miguel, A. (Ed.). Entre Estado y Cosmópolis. Derecho y justicia en un mundo global, Madrid, Editorial Trotta, 2014], pp. 179-212.

Añón Roig, María "Principio antidiscriminatorio y determinación de la desventaja". Isonomía, 39 (2013), pp. 127-157.

Atria, Fernando "Sobre la función judicial en tiempos de constitucionalismo global”. [En: Aguilar, G. (Coord.). Diálogo entre jurisdicciones. Santiago, Librotecnia, 2014], pp. 467-484.

Atria, Fernando La forma del derecho, Madrid, Marcial Pons, 2016, 500 pp.

Francisco Barbosa, "Los límites a la doctrina del margen nacional de apreciación en el Tribunal Europeo y la Corte Interamericana de Derechos Humanos: intervención judicial en torno a ciertos derechos de las minorías étnicas y culturales". Revista Derecho del Estado, 26 (2011), pp. 107-135.

Barrère María y Morondo, Dolores "Subordinación y discriminación interseccional: elementos para una teoría del derecho antidiscriminatorio". Anales de la Cátedra Francisco Suárez, 45 (2011), pp. 15-42.

Benavides María, "El consenso y el margen de apreciación en la protección de los derechos humanos". Iut et Praxis, 15 (2009), pp. 295-310.

Caicedo, Natalia "La interpretacion de los derechos y libertades en sociedades culturalmente diversas". [En: Caicedo, N. y Moya, D. (Coords.). Diversidad cultural e interpretación de los derechos. Madrid, Centro de Estudios Políticos y Constitucionales, 2015], pp. 21-62.

Cançado Trindade, Antonio El Derecho Internacional de los Derechos Humanos en el siglo XXI, Santiago de Chile, Editorial Jurídica, 2006, 546 pp.

Carmona, Encarna "La prohibición de la discriminación. Nuevos contenidos". [En: García, J. y Santolaya, P. (Coords.). La Europa de los Derechos. El Convenio Europeo de Derechos Humanos. Madrid, Centro de Estudios Políticos y Constitucionales, 2014], pp. 587-612.

Contreras, Pablo "National Discretion and International Deference in the Restriction of Human Rights: A Comparison Between the Jurisprudence of the European and Inter-American Court of Human Rights". Northwestern Journal of International Human Rights, 1 (2012), pp. 28-82.

Cook Rebecca y Cusack, Simone Estereotipos de género: perspectivas legales transnacionales, Colombia, Profamilia, 2010, 291 pp. 
Denninger, Erhard "Derecho y procedimiento jurídico como engranaje en una sociedad multicultural" [En: Gutiérrez Gutiérrez, I. (Ed. y Trad.). Derecho constitucional para la sociedad multicultural, Madrid, Trotta, 2007], pp. 27-50.

Dworkin, Ronald Los derechos en serio, Barcelona, Ariel, 1984, 508 pp.

Faúndez, Héctor El Sistema Interamericano de Protección de los Derechos Humanos, Costa Rica, Instituto Interamericano de Derechos Humanos, 2004, 1085 pp.

Fraser, Nancy "La justicia social en la era de la política de la identidad: Redistribución, reconocimiento y participación”. [En: Fraser, N. y Honneth, A. ¿Redistribución o reconocimiento? Madrid, Morata, 2006], pp. 17-88.

Gajardo Falcón, Jaime "Comentario de la STEDH Aksu v. Turquía de 15 de marzo de 2012. Ampliación del concepto de víctima e interpretación contextualizada de los derechos humanos". Revista Jurídica de la Universidad Autónoma de Madrid, 30 (2014), pp. 273-286.

Gajardo Falcón, Jaime "La prohibición del velo integral en los espacios públicos y el margen de apreciación de los Estados. Un análisis crítico de la sentencia del TEDH de 01.07.2014, S.A.S. c. Francia, 43835/11”. Revista de Derecho Comunitario Europeo, 51 (2015), pp. 769-783.

Gargarella Roberto, "Justicia penal internacional y deliberación democrática. El caso Gelman”. [En: Santolaya, P. y Wences, I. (Coords.). La América de los Derechos, Madrid, Centro de Estudios Políticos y Constitucionales, 2016], pp. 409-433.

Gutiérrez Gutiérrez, Ignacio "Introducción: Derecho Constitucional para la sociedad multicultural". [En: Gutiérrez Gutiérrez, I. (Ed.). Derecho constitucional para la sociedad multicultural, Madrid, Trotta, 2007], pp. 9-25.

Grimm, Dieter "Multiculturalidad y derechos fundamentales" [En: Gutiérrez Gutiérrez, I. (Ed. y Trad.). Derecho constitucional para la sociedad multicultural. Madrid, Trotta, 2007], pp. 51-69.

Habermas, Jürgen The Inclusion of the Other. Cambridge, Polity, 1998, 300 pp. Hart Ely, John Democracy and Distrust. Cambridge, Harvard University Press, 1980, 268 pp.

Iglesias Vila, Marisa "La aplicación del CEDH desde una concepción política de los derechos humanos: algunas claves interpretativas". [En: Arcos, F. (Ed.). La justicia y los derechos en un mundo globalizado, Madrid, Dykinson, 2016], pp. 167-190.

Kymlicka, Will "Comments on Shachar and Spinner-Halev: An Update from the Multiculturalism Wars”. [En: Joppke, Ch. y Lukes, S. (Eds.). Multicultural Questions, Oxford, Oxford University Press, 1999], pp. 112-129. 
La Barbera, MaríaCterina "El enfoque de la interseccionalidad aplicado a las políticas para la erradicación de la mutilización femenina”. [En: García, F. y Kressova, N. (Coords.). Actas del Congreso Internacional sobre Migraciones en Andalucía, Granada, Instituto de Migraciones, 2011], pp. 2191-2193.

Martínez Estay, José "Subsidiariedad y margen de apreciación en las relaciones derecho nacional-derecho supranacional. Consideraciones para el diálogo entre la Corte Interamericana de Derechos Humanos y los tribunales nacionales". [En: Nogueira, H. (Coord.). La protección de los Derechos Humanos y fundamentales de acuerdo a la Constitución y el Derecho Internacional de los Derechos Humanos, Santiago de Chile, Librotecnia, 2014], pp. 307-344.

Núñez, Manuel "Sobre la doctrina del margen de apreciación nacional. La experiencia latinoamericana confrontada y el thelos constitucional de una técnica de adjudicación del derecho internacional de los derechos humanos". [En: Núñez Poblete, M. y Acosta, P. (Eds.). El margen de apreciación en el Sistema Interamericano de Derechos Humanos: proyecciones regionales y nacionales, México, UNAM, 2012], pp. 3-49.

Paúl, Álvaro "Decision-Making Process of the Inter-American Court: an Analysis Prompted by the 'In Vitro Fertilization' Case”. ILSA Journal of International \& Comparative Law, 1 (2014), pp. 87-130.

Pentassuglia, Gaetano Minority Groups and Judicial Discourse in International Law, Leiden, Martinus Nijhoff, 2009, 276 pp.

Perry, Michael Toward a Theory of Human Rights: Religion, Law, Courts, Cambridge, Cambridge University Press, 2007, 270 pp.

Popelier Patricia y Van de Heyning, Catherine "Subsidiarity Post-Brighton: Procedural Rationality as Answer?". Leiden Journal of International Law, 30 (2017), pp. 5-23.

Roa, Jorge "Pluralismo jurídico y mecanismos de coordinación entre los sistemas de justicia indígena y el sistema nacional de justicia en Colombia". Revista Derecho del Estado, 33 (2014), pp. 101-121.

Sordo, Tania "Ella se lo buscó. Estereotipos de género en el Estado mexicano: Sentencia Campo Algodonero". XV Premio SIEM de Investigación feminista "Concepción Gimeno de Flaquer", Universidad de Zaragoza, 2012, pp. 1-25.

Sordo, Tania "Los estereotipos de género como obstáculos para el acceso de las mujeres a la justicia”. [En: Ética judicial e igualdad de género". México, Suprema Corte de Justicia de la Nación, 2014], pp. 319-352.

Tully, James "The Practice of Law-Making and the Problem of Difference: An Introduction to the Field". [En: Payrow, O. (Ed.). Multiculturalism and Law. Cardiff, University of Wales Press, 2007], pp. 19-41. 
Torres Zúñiga, Natalia "Control de normas constitucionales por la Corte Interamericana de Derechos Humanos: Subsidariedad, deferencia e impacto en la teoría del cambio constitucional". [En: Saiz, A. (Dir.). Diálogos Judiciales en el Sistema Interamericano de Derechos Humanos, Valencia, Tirant lo blanch, 2016], pp. 90-126.

Verdugo Sergio y García, José "Radiografía al sistema interamericano de derechos humanos". Revista Actualidad Jurídica, 25 (2012), pp. 175-216.

Waldron, Jeremy Derechos y desacuerdos, Barcelona, Marcial Pons, 2005, 395 pp.

Young, Iris Inclusion and Democracy. New York, Oxford, 2000, 304 pp. 
